\title{
Clinical Spectrum and Subtype Distribution Lymphoma : A Single Center, Hospital Based Analysis
}

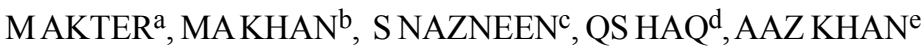

Summary:

Introduction: Lymphoma is a heterogeneous group of hematological malignancy that varies in different subtypes according to geography, age, race and ethnicity. In this study, the different subtype of lymphoma according to WHO lymphoid tumor classification and clinical features of Non Hodgkins lymphoma will be analyzed and discussed at a tertiary care Hematology center.

Objectives: Our aims and objective is to observe and share the single center experience of specifically Non-Hodgkin lymphoma and to contribute in formation of national lymphoma registry in future to enhance the care of potentially curable lymphomas.

Materials and Method: A retrospective analysis of 226 diagnosed lymphoma cases were conducted at DMCH Hematology center from January 2016 to September 2017( total 21 months period). Data were reviewed and analyzed using simple frequency and percentage. Protocol was approved by institutional ethical review board (IRB) of DMCH.

Result: The mean age of NHL is 43 (12-90) years with majority of patients were in 31-55 years age group and M:F is 3:1. The mean age of $\mathrm{HL}$ is 30 (range 4-60) years without bimodal peak observed. Mixed cellularity classical HL were found higher than nodular sclerosis $\mathrm{HL}$.

Introduction:

Lymphoid neoplasm originating from either $\mathrm{B}$ or $\mathrm{T}$ lymphocytes is heterogeneous both biologically and clinically. The incidence of lymphoma is increasing all

a. Dr. Mafruha Akter, Assistant Professor, BMT unit, Hematology Department, Dhaka Medical College, ospital $(\mathrm{DMCH})$

b. Prof. Dr. Mohiuddin Ahmed Khan, Professor, Hematology Department, Dhaka Medical College Hospital (DMCH).

c. Dr. Shahela Nazneen, Assistant Professor, Hematology Department, Dhaka Medical College Hospital (DMCH).

d. Dr. Qauzi Smita Haq, Hematology Department, Dhaka Medical College Hospital (DMCH)

e. Dr. Abdullah Al Zubayer Khan, Registrar, BMT unit, Department Of Hematology, Dhaka Medical College Hospital (DMCH)

Address of Correspondence: Dr Mafruha Akter, Assistant Professor, BMT unit, Hematology Department, Dhaka Medical College, ospital (DMCH), Email: mafruha673@gmail.com Phone: +8801912112724

Received: 5 July, 2018

Accepted: 10 July, 2019
Majority of NHL were B cell NHL (86.25\%) and remaining were T cell NHL (13.75\%).

The most common variant found was aggressive diffuse large B cell lymphoma(DLBCL) (48\%), followed by peripheral T cell lymphoma PTCL ( 13\%) and very aggressive lymphoblastic lymphoma (LBL) (11\%), low grade follicular Lymphoma (11\%) and others ( 17\%).

$70 \%$ NHL had nodal presentation and $30 \%$ had extra nodal involvement with GIT and CNS most commonly involved. Extra nodal presentations were more observed in DLBCL and $L B L$. Majority (75\%) of NHL presented at advanced stage with B symptoms observed in $86 \%$ and variable IPI score. In DLBCL cell of origin was detected as non-GCB in 25(41\%), GCB in 04( 6\%) and unclassifiable in 03(5\%) cases according to Han's algorithm, and cell of origin was not detected in remaining cases.

Conclusion: This is a small scale retrospective study, this can lead raising awareness of doing large scale national data registry for various lymphoma patients. The thorough clinical and diagnostic information about lymphoma is necessary for better management and outcome.

Key words: HL, NHL, PTCL, DLBCL, GCB, non GCB, LBL.

(J Bangladesh Coll Phys Surg 2020; 38: 23-28)

DOI: http://dx.doi.org/10.3329/jbcps.v38i1.44685

over the world specially in western countries. A multicenter retrospective study reported that $\mathrm{NHL}$ and HL comprises $16.9 \%$ and $3.9 \%$ among the hematological malignancies over a 5 years study period in Bangladesh. ${ }^{1}$ Moreover different epidemiologic studies have shown considerable differences in the distribution of lymphoma subtypes between Asian and western populations. ${ }^{2,3,4}$ Over the past few decades the classification of NHL has emergently being changed. World Health Organization (WHO) revised the consensus classification of lymphoma in 2001 and 2008. Finally WHO most recently updated the 2016 version that encompassed morphology, immunophenotype, biology of tumor and gene expression of lymphoma. ${ }^{5}$ The therapy and prognosis of HL and NHL depend on the subtype, stage and associated co-morbid conditions. However there is lack of potential data of subtype distribution and clinical presentation pattern of both Hodgkins and non Hodgkin lymphoma in Bangladesh. The purpose of 
this paper is to observe and share the single centre experience of subtype distribution of all lymphoma and clinical features of only NHL .

\section{Materials and Method:}

A retrospective analysis of histopathologic and immunohistochemistry profiles and clinical features of different pattern of diagnosed 226 lymphoma cases were conducted who attended at DMCH Hematology center from January 2016 to September 2017( total 21 months period). Data were analyzed and reviewed and expressed as frequency and simple percentage. The protocol of this study was approved by the institutional ethical review board ( IRB) of Dhaka Medical College Hospital.

\section{Result:}

A total 226 Lymphoma patients were included in this study who attended at Hematology department of DMCH from different parts of the country. Out of total 226 lymphoma cases 160 patients were NHL and 66 were HL. The mean age of HL is 30 (range 4-60) years without bimodal peak observed. Among the HL, 24 (36.6\%) were mixed cellularity, $5(7 \%)$ were nodular sclerosis and in $32(48 \%)$ cases histopathologic subtype were not mentioned, rather reported as classical HL. Nodular lymphocyte predominant HL(NLPHL) and lymphocyte depleted variety were observed in 5 cases $(3 \& 2$ respectively). All these $\mathrm{HL}$ cases were being confirmed by immunohistochemistry (IHC).

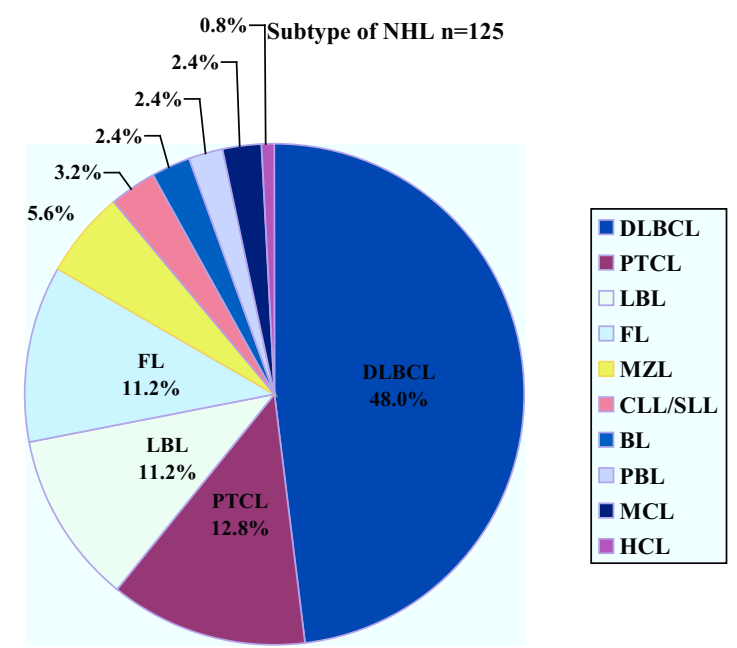

Fig.-1: Different subtype distribution of NHL according to WHO classification.
Among the 160 NHL patients, 138 (86.25\%) cases were B cell type and remaining 22(13.75\%) cases were T cell type.

Median age at diagnosis was 43(12-90) years and M:F ratio was 3: 1 for all NHL patients. Among the $160 \mathrm{NHL}$ patients, 35 patients were not been able to be evaluated due to lack of proper data and lack of enough immunohistochemitry profile. Finally 125 NHL patient's clinical parameters, histopathology, IHC and other investigations were evaluated. The most common variant was found is aggressive DLBCL (60/48.38\%), followed by PTCL 16(12.8\%), very aggressive lymphoblastic lymphoma (LBL) 14(11.2\%), low grade follicular lymphoma 14(11.2\%) and others 21 (16.42\%) comprising marginal zone lymphoma, Mantle cell lymphoma, chronic lymphocytic leukemia/small lymphocytic lymphoma,

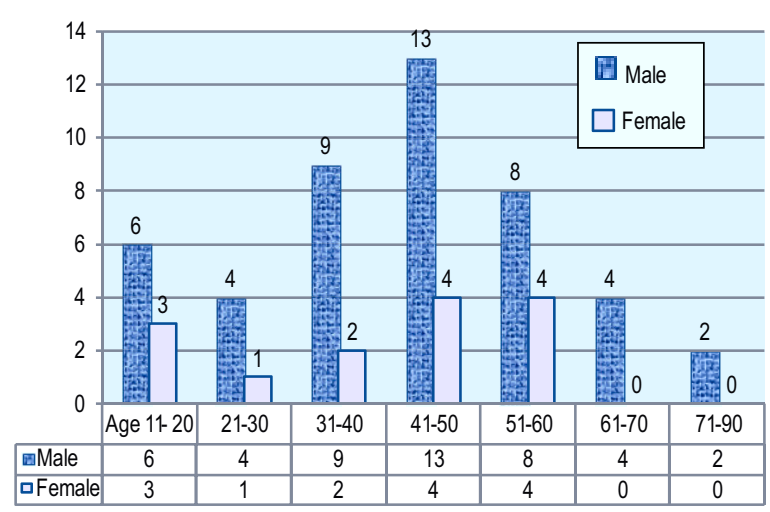

Fig 2: Age and sex distribution of $\operatorname{DLBCL}(n=60)$

Hairy cell leukemia, plasmablastic lymphoma and Burkitts Lymphoma.

Most of the DLBCL (45/75\%) patients presented with advanced stage III/IV disease, remaining $25 \%$ were detected at early I/II stage disease. Majority of these DLBCL patients ( $86 \%$ ) had B symptoms at diagnosis. While almost all patients of lymphoblastic lymphoma (LBL) and PTCL had III/IV disease. Forty two (70\%) patients of DLBCL had nodal presentation and remaining $18(30 \%)$ had extranodal presentation with or without nodal involvement, comprising most common extranodal site is GIT and CNS. Other rare extranodal sites were breast, paranasal sinus, soft palate, urinary bladder, tonsil, shoulder and testes. Very few of $\operatorname{DLBCL}(06 / 10 \%)$ had bone marrow involvement at 


\section{Table-I}

Clinical presentation of major subtype of $N H L$

Patient profile Number / percentage

\begin{tabular}{|c|c|c|c|c|}
\hline & $\begin{array}{c}\text { DLBCL } \\
n=60\end{array}$ & $\begin{array}{l}\text { PTCL } \\
\mathrm{n}=16 \\
\end{array}$ & $\begin{aligned} \text { LBL } n & =14 \\
n & =14\end{aligned}$ & FL \\
\hline \multicolumn{5}{|l|}{ Age in Years: } \\
\hline$<60$ & 50 & 13 & 1407 & \\
\hline$>60$ & 10 & 03 & 0007 & \\
\hline Range & $(12-90)$ & $(18-70)$ & $(14-28)$ & $(30-69)$ \\
\hline \multicolumn{5}{|l|}{ Sex } \\
\hline Male & 45 & 14 & 1010 & \\
\hline Female & 15 & 02 & 0404 & \\
\hline M:F ratio & $3: 1$ & $7: 1$ & $2.5: 12.5: 1$ & \\
\hline B symptoms: & $52(86 \%)$ & $15(93 \%)$ & $14(100 \%)$ & $07(50 \%)$ \\
\hline \multicolumn{5}{|c|}{ Fever /Night sweats /weight loss } \\
\hline \multicolumn{5}{|l|}{ Lymph node enlargement } \\
\hline - Cervical \pm Supraclavicular & 50 & 13 & 0911 & \\
\hline - $\quad$ Axillary & 20 & 08 & 06 & \\
\hline - Mediastinal & 12 & 05 & 08 & \\
\hline - Abdominal & 45 & 10 & 06 & \\
\hline - Inguinal & 49 & 10 & 0408 & \\
\hline Hepatomegaly & 20 & 05 & 0203 & \\
\hline Splenomegaly & 43 & 04 & 0503 & \\
\hline Bone marrow infiltration & 10 & 02 & 0405 & \\
\hline Extra nodal site & GIT -06 & & CNS-02 & GIT-01 \\
\hline CNS-04 & & Breast-01 & & \\
\hline Breast-02 & & Pericardium & & \\
\hline Tonsil-02 & & -01 & & \\
\hline Para nasal sinus -02 & & Pleura-02 & & \\
\hline \multicolumn{5}{|l|}{ Para nasal sinus -01} \\
\hline \multicolumn{5}{|l|}{ Shoulder Joint -01Leg -01 } \\
\hline AIHA & 02 & & 02 & \\
\hline ITP 02 & 01 & - & 02 & \\
\hline \multicolumn{5}{|l|}{ SLDH } \\
\hline - $\quad$ Raised & 23 & 10 & 1103 & \\
\hline - $\quad$ Normal & 20 & 06 & -09 & \\
\hline - $\quad$ Not documented & 17 & & 0302 & \\
\hline ECOG & d"2 & 4218 & 06100410 & 1103 \\
\hline \multicolumn{5}{|l|}{ - $\quad>2$} \\
\hline Staging & e’III/IV & 45 & 1013 & 09 \\
\hline - $\quad \leq \mathrm{II}$ & 15 & 06 & 0105 & \\
\hline
\end{tabular}


Table-2

$H L$ and NHL distribution according to Immunohistochemistry $n=226$

\begin{tabular}{|c|c|c|c|}
\hline $\mathrm{B}$ cell $\mathrm{NHL}=97$ & Number $(\%)$ & $\mathrm{T}$ cell $\mathrm{NHL}=28$ & Number $(\%)$ \\
\hline DLBCL & $60(48 \%)$ & PTCL & $16(12.8 \%)$ \\
\hline GCB & 25 & · PTCLNOS & 13 \\
\hline Non GCB & 04 & · Angioimmunoblastic & 02 \\
\hline Unclassifiable & 03 & · Anaplastic ALK neg & 01 \\
\hline Not documented & 28 & $\cdot$ & \\
\hline FL(Follicular Lymphoma) & $14(11.2 \%)$ & T Lymphoblastic & $12(9.6 \%)$ \\
\hline MZL(Marginal Zone Lymphoma) & $07(5.6 \%)$ & Lymphoma & \\
\hline CLL/SLL(Chronic Lymphocytic & $04(3.2 \%$ & Hodgkins Lymphoma & 66 \\
\hline Leukemia/ Small Lymphocytic & & · Mixed cellularity cHL & $24(36.36 \%)$ \\
\hline \multicolumn{4}{|l|}{ Lymphoma) } \\
\hline Nodular sclerosis cHL & $05(7.5 \%)$ & & \\
\hline PBL( Plasmablastic Lymphoma) & $03(2.4 \%)$ & · Lymphocyte depleted & \\
\hline MCL( Mantle cell Lymphoma) & $03(2.4 \%)$ & - $\mathrm{cHL}$ & 02 \\
\hline Burkitts L & $03(2.4 \%)$ & · NLPHL & 03 \\
\hline \multirow{2}{*}{$\begin{array}{l}\text { B LBL (B Lymphoblastic Lymphoma) } \\
\text { but }\end{array}$} & $02(1.6 \%)$ & - Histopath+IHC HL & $32(48.48 \%)$ \\
\hline & without subty & & \\
\hline HCL( Hairy cell leukemia) & $01(0.8 \%)$ & mentioned & \\
\hline NHL by histopath and limited panel IHC & 35 & & \\
\hline
\end{tabular}

diagnosis evident by morphology and or IHC. International prognostic index (IPI) score for DLBCL was variable having 27 (45\%) had IPI score 2-3( intermediate) and 25(41.6\%) had high IPI 4-5 score, $08(13 \%)$ had low IPI score $0-1$. DLBCL cell of origin was detected as non GCB in 25(41\%), GCB in 04( 6\%) and unclassifiable in $03(5 \%)$ cases according to Han's algorithm, although $48 \%$ cases were not been able to be classified due to lack of documentation of CD10, BCL6 and MUM1 markers.

PTCL patients had similar fashion of clinical features. Most patients of the very aggressive $\mathrm{T}$ lymphoblastic lymphoma ( T LBL) had mediastinal involvement.

Different modalities of chemotherapies were received in various lymphoma according to disease status, stage and their financial ability.

\section{Discussion:}

Subtype distribution of lymphoma varies strikingly by geographic, demographic, etiologic, ethnic and environmental factors. The heterogeneity of the lymphoma depends on these factors and presentation differs in different subtype. In this study we present the patterns and distribution of lymphoma according to WHO classification in a tertiary hospital based data in Bangladesh.
According to the SEER data HL made up of $11 \%$ of all lymphomas ${ }^{6}$ and nodular sclerosis HL are more frequent than mixed cellularity ( $70 \%$ Vs $25 \%$ ) in western countries. $^{7,8}$ In our 21 months hospital based data showed that higher HL (29\%) patients were enrolled among all lymphoma with predominantly (36.6\%) mixed cellularity ( MC) cHL followed by nodular sclerosis (NS) cHL only $7 \%$. It is to be mentioned that in half of cases (48\%) histologic subtype were not mentioned, but all those cases were being confirmed by IHC. In a large retrospective study of China found that $73.3 \%$ of $\mathrm{MC}$ cHL had EBV. ${ }^{9}$ Although EBV was not confirmed in all cases in our study but childhood EBV associated infection may have contribution in high frequency of MC CHL in Asian country. Another difference in demography of HL in our study that we did not find any bimodal age distribution which was found in Europe and North America. Japan also shows that a single peak age of HL in elderly people. ${ }^{10}$ Nakatsuka and Aozasa have pointed out that the bimodal age curve might be formed by the different peak ages of the two main subtypes, MC-CHL (later years) and NS-CHL (young adults). ${ }^{11}$

NHL is one of the leading hematological malignancy in adult worldwide. The mean age in western and Asian 
countries is between 50 to 60 years. ${ }^{12,13}$ In our study we found an earlier median age at diagnosis 43( 12-90) years for all NHL patients and M:F ratio is 3: 1 that is almost similar to western countries ratio.

In our study, B-cell lymphoma comprises $86.25 \%$ and remaining $13.75 \%$ were T-cell lymphomas very close to that of other studies in India ${ }^{4}$ but differs that of a large study in China ${ }^{9}$ that reported higher percentage (30.2\%) of PTCL and NK cell lymphoma. Researchers has suggested that environmental factors including EBV infection as well as exposure to pesticides and chemical solvents were strongly associated with the higher frequency of T-cell NHL in China considering the agricultural area. ${ }^{9,14}$

According to clinical behavior most of the NHL in this current study were aggressive (65\%), followed by indolent $(21 \%)$ and very aggressive (14\%). With regards to NHL subtype distribution, DLBCL continues to be the dominant histology comprising $30-35 \%$ of all NHL cases worldwide and this proportion has been stable over the years. ${ }^{15}$ Similarly in our study the most common variant NHL found was DLBCL although the percentage is higher ( $48.38 \%$ ) than other studies. Next common variety lymphoma were PTCL, followed by lymphoblastic lymphoma and follicular lymphoma. Other non-Hodgkins lymphomas found scattered were marginal zone lymphoma, mantle cell lymphoma, chronic lymphocytic leukemia/small lymphocytic lymphoma, hairy Cell leukemia, plasmablastic lymphoma and Burkitts lymphoma.

Similar result was observed in an Indian study where higher frequency of DLBCL (50.2\%) was followed by follicular lymphoma (13.2\%), ${ }^{16}$ though our study had lower frequency of follicular type (11\%). However, this trend of low frequency of follicular type (1.8\%) was observed in Asian studies too. ${ }^{13}$

The gene expression profile (GEP) in research has classified the prognosis of DLBCL according to cell of origin. Based on Han's algorithm ${ }^{17}$ in our study DLBCL cell of origin was detected as non GCB in $41 \%$ (25), GCB in 6\% (04) \& unclassifiable in 5\% (03) cases, although $48 \%$ cases were not been able to be classified due to lack of proper immuno-histochemistry profile whereas much higher percentage (79\%) non GCB DLBCL was reported in Chinese patients. ${ }^{18}$
Most of the DLBCL patients (70\%) had lymph node enlargement in different site including cervical, axilla, abdominal and inguinal region. About $30 \%$ patients had extranodal disease with or without nodal involvement, comprising most common extranodal site was GIT and CNS and rare sites were breast, para nasal sinus, shoulder and testes. Very few of DLBCL $(10 \%)$ had BM involvement at initial diagnosis. Two third of DLBCL presented with advanced stage and $86 \%$ having systemic B symptoms in our study comparatively higher than South India (23.9\%) and Pakistan (36.7\%). ${ }^{19,20}$

PTCL patients had similar fashion of clinical features. Patients with LBL had mostly mediastinum as extranodal site of involvement.

Staging PET scan is not routinely done at our center due to lack of financial support of patient group, staging CT scan is done in almost every cases.

HL and NHL patients are being treated in this center with various chemotherapy protocols according to their disease status, staging and prognosis. Autologous stem cell transplant is available for the relapse and refractory lymphoma patients who are chemo-sensisitive with salvage chemotherapy.

Limitation:

Our study has some limitations. This is a small scale study in a short period that might not represent the national data. The pathological reports were not reviewed due to lack of central reference lab and limited number of hematopathologist in our country . Immunohistochemistry ( IHC) facilities are available only in very limited laboratories and sometimes the reports are not comprehensive to reach a diagnosis. There is lack of institutional policy of keeping medical records of patients, so detail clinical information of Hodgkins lymphoma was not included in this study. However this study is able to reflect the clinicopathologic pattern and distribution of lymphoma in a single center .

\section{Conclusion}

The Non Hodgkin Lymphoma is a common hematologic malignancy worldwide including Bangladesh. But there is scarcity of national data representing incidence, subtype and clinical spectrum of lymphoma that is necessary for proper management and better outcome . Although this is a small scale retrospective study, this can lead raising awareness of doing large scale national 
data registry for lymphoma patients thus will be helpful to deliver standard of care treatment facilities to lymphoma patients as well as Autologous stem cell transplant for relapsed lymphoma.

\section{References:}

1. Hossain M, Iqbal M, Ahmed Khan M, Rabbani M, Khatun $\mathrm{H}$, Munira S, et al. Diagnosed hematological malignancies in Bangladesh - A retrospective analysis of over 5000 cases from 10 specialized hospitals. BMC cancer. 2014; Vol. 14: 438-40.

2. Anderson JR, Armitage JO, Weisenburger DD. Epidemiology of the non- Hodgkin's lymphomas: distributions of the major subtypes differ by geographic locations. NonHodgkin's Lymphoma Classification Project. Ann Oncol 1998; 9:717-20.

3. Fisher SG, Fisher RI. The epidemiology of non-Hodgkin's lymphoma. Oncogene. 2004;23:6524-34.

4. Devi AA, Sharma TD, Singh YI, Sonia H. Clinicopathological profile of patients with non- hodgkin's lymphoma at a regional cancer center in Northeast India. J Sci Soc 2017;44:140-4.

5. Steven H, Swerdlow, Elias Campo, Stefano A. Pileri, Nancy Lee Harris et al. The 2016 revision of the World Health Organization classification of lymphoid neoplasms. Blood. 2016; 127:2375-90.

6. Jemal A, Siegel R, Xu J, Ward E: Cancer Statistics, 2010. CA Cancer J Clin. 2011; 60:277-300.

7. Jaffe ES, NL H, Stein H, Vardiman JW: WHO Classification: Pathology and Genetics of Tumors of Haematopoietic and Lymphoid Tissues Lyon: IARC; 2001

8. Morton LM, Wang SS, Devesa SS, Hartge P, Weisenburger DD, Linet MS: Lymphoma incidence patterns by WHO subtype in the United States, 1992-2001. Blood. 2006; 107:265-76.

9. Yang Q-P, Zhang W-Y, Yu J-B, et al. Subtype distribution of lymphomas in Southwest China: Analysis of 6,382 cases using WHO classification in a single institution. Diagnostic Pathology. 2011;6:77.
10. Aoki R, Karube K, Sugita Y, Nomura Y, Shimizu K, Kimura $\mathrm{Y}$ et al. : Distribution of malignant lymphoma in Japan: analysis of 2260 cases, 2001-2006. Pathol Int 2008; 58:174-82.

11. Nakatsuka S, Aozasa K: Epidemiology and pathologic features of Hodgkin lymphoma. Int J Hematol 2006; 83:391-97.

12. García Zueco JC, Delgado MP, Giraldo MP, Perella M, Torres M, Valero MI, et al. Non-Hodgkin's lymphomas. I. Clinico-biological features of 307 cases. Sangre (Barc). 1992;37:331-36.

13. Yoon SO, Suh C, Lee DH, Chi HS, Park CJ, Jang SS, et al. Distribution of lymphoid neoplasms in the republic of Korea: Analysis of 5318 cases according to the World Health Organization classification. Am J Hematol. 2010;85: 760-64.

14. Xu JX, Hoshida Y, Yang WI, Inohara H, Kubo T, Kim GE, et al: Life-style and environmental factors in the development of nasal NK/T-cell lymphoma: a case-control study in East Asia. Int J Cancer 2006; 120:406-10.

15. Mohammed Al-Hamadani, Thomas M. Habermann, James R. Cerhan, William R. Macon, Matthew J. Maurer and Ronald S. Go. Non-Hodgkin lymphoma subtype distribution, geodemographic patterns, and survival in the US: A longitudinal analysis of the National Cancer Data Base from 1998 to 2011. Am. J. Hematol. 2015, 90:790-95.

16. Sahni CS, Desai SB. Distribution and clinicopathologic characteristics of non-Hodgkin'slymphoma in India: A study of 935 cases using WHO classification of lymphoid neoplasms (2000). Leuk Lymphoma. 2007;48:122-33.

17. Hans C, Weisenburger D, Greiner T, Gascoyne R, Delabie J, Ott $\mathrm{G}$, et al: Confirmation of the molecular classification of diffuse large B-cell lymphoma by immunohistochemistry using a tissue microarray. Blood. 2004; 103:275-82.

18. Chen Y, Han T, Iqbal J, Irons R, Chan W, Zhu X, et al. Diffuse Large B-Cell Lymphoma in Chinese Patients: Immunophenotypic and Cytogenetic Analyses of 124 Cases. Am J Clin Pathol. 2010;133:305-13.

19. Hingorjo MR, Syed S. Presentation, staging and diagnosis of lymphoma: A clinical perspective. J Ayub Med Coll Abbottabad. 2008;20:100-03.

20. Neeravari VS, Bannigidad D. Clinical spectrum of nonHodgkin lymphoma: A hospital based study of 410 Cases. Ann Appl Bio Sci. 2016;3:89-93. 